Rapid Reviews COVID-19

\title{
Reviews of "Virtual reality exercise to help COVID patients with refractory breathlessness"
}

Giuseppe Riva ${ }^{1}$, Fabrizio Stasolla ${ }^{2}$

${ }^{1}$ Catholic University of Sacred Heart, ${ }^{2}$ Università Telematica Giustino Fortunato

Published on: Jan 25, 2022

DOI: $10.1162 / 2$ e3983f5.1f1fcf9c

License: Creative Commons Attribution 4.0 International License (CC-BY 4.0). 
To read the original manuscript, click the link above.

Summary of Reviews: This preprint evaluates whether a virtual reality-based rehabilitation program could improve breathing comfort in patients recovering from COVID-19 and found this iVR-based DTx treatment protocol was effective. Reviewers rated the study as potentially informative but confounded.

\section{Reviewer 1 (Giuseppe Riva) |}

\section{Reviewer 2 (Fabrizio Stasolla)}

$$
\begin{aligned}
& \text { RR:C19 Strength of Evidence Scale Key. } \\
& \text { प्रमप = Misleading }
\end{aligned}
$$

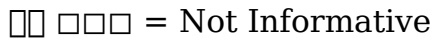

$$
\begin{aligned}
& \text { प्रा पि = Potentially Informative }
\end{aligned}
$$

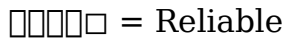

$$
\begin{aligned}
& \text { प्राप्र = Strong }
\end{aligned}
$$

To read the reviews, click the links below. 